\title{
Microbial hitchhiking: how Streptomyces spores are transported by motile soil bacteria
}

\author{
Alise R. Muok $\mathbb{B}^{1,2} \cdot$ Dennis Claessen $\mathbb{B}^{1,2} \cdot$ Ariane Briegel $\mathbb{C}^{1,2}$
}

Received: 24 August 2020 / Revised: 19 February 2021 / Accepted: 25 February 2021 / Published online: 15 March 2021

(c) The Author(s) 2021. This article is published with open access

\begin{abstract}
Streptomycetes are sessile bacteria that produce metabolites that impact the behavior of microbial communities. Emerging studies have demonstrated that Streptomyces spores are distributed through various mechanisms, but it remains unclear how spores are transported to their preferred microenvironments, such as plant roots. Here, we show that Streptomyces spores are capable of utilizing the motility machinery of other soil bacteria. Motility assays and microscopy studies reveal that Streptomyces spores are transported to plant tissues by interacting directly with the flagella of both gram-positive and gramnegative bacteria. Genetics experiments demonstrate that this form of motility is facilitated by structural proteins on the spore coat. These results demonstrate that nonmotile bacteria are capable of utilizing the motility machinery of other microbes to complete necessary stages of their lifecycle.
\end{abstract}

\section{Introduction}

Bacteria belonging to the genus Streptomyces are an integral component of diverse ecosystems and are well-known to produce chemically diverse metabolites, including the vast majority of all clinically relevant antibiotics [1, 2]. Soildwelling Streptomycetes, such as Streptomyces coelicolor $(S c)$, colonize plant roots and provide the associated plant protection from potential phytopathogens through antibiotic secretion [1]. The symbiosis of Streptomycetes with their plant hosts has been shown to improve plant health and productivity, and thereby provides a potential sustainable solution to increase crop yields [2-5]. The lifecycle of Streptomycetes is complex and involves stages of aerial hyphae formation on the soil surface to produce spores, and spore germination on plant roots to produce filamentous colonies [1]. Immotile Streptomyces bacteria distribute their

Supplementary information The online version contains supplementary material available at https://doi.org/10.1038/s41396021-00952-8.

Ariane Briegel

a.briegel@biology.leidenuniv.nl

Institute for Biology, Leiden University, Leiden, The Netherlands

2 Centre for Microbial Cell Biology, Leiden University, Leiden, The Netherlands spores over long distances through attachment to insects and nematodes, but it is unclear how they relocate over short distances to their preferred microenvironments such as plant root systems [6, 7].

While Streptomyces are nonmotile, many other soil microbes, such as Bacillus subtilis (Bs) and Pseudomonas fluorescens $(P f)$, are motile and can move through their environment by regulating the rotation of flagella [8]. On solid surfaces, flagellar rotation enables cell swarming. During swarming, cells are densely packed and continuously move outward toward unoccupied areas. In liquid media, flagella enable cell swimming where the cells move independently and can rapidly change swimming direction. In addition to flagellar motility, some bacteria can also move through a passive diffusion on surfaces called sliding [8]. Sliding does not involve flagella but occurs when cells are pushed through the forces generated by the outward growing colony.

Recent reports have revealed that microbe transport by inter-species interactions can occur between motile and immotile microbes [9]. These studies demonstrate that intermicrobial transport occurs among organisms natively found on abiotic surfaces [10,11], plant surfaces [12], and within the soil $[13,14]$. In some instances, the transportation of human pathogens are facilitated on abiotic surfaces, including nonmotile Staphylococcal species that directly adhere to their mobile partners [10], Aspergillus fumigatus (Af) spores that interact with the flagella of motile bacteria 
[14], various nonmotile human microbiome bacteria that are carried by Capnocytophaga gingivalis [15], and Legionella pneumophila that is transported internally by their amoebae hosts [16].

Here, we demonstrate that spores of the sessile Streptomycetes, such as $S c$, are transported by $B s$ to their preferred microenvironment. $S c$ and $B s$ are both soildwelling bacteria that utilize plant root exudate as a nutritive source [1, 17]. Using microscopy methods, motility assays, and genetics approaches, we demonstrate that $B s$ transports $S c$ spores via direct attachment to $B s$ flagella, a mode of transportation we call "hitchhiking". Hitchhiking is dependent on the conserved rodlin proteins, which form a fibrous outer layer on the spore coat of almost all Streptomycetes, but with a hitherto unclear function $[18,19]$. These results exemplify that nonmotile bacteria are capable of utilizing the motility machinery of other microbes to occupy advantageous environments, and that this mode of transport may be widespread in nature.

\section{Results}

\section{Bacillus subtilis disperses Streptomyces coelicolor spores}

Transportation of $S c$ spores by $B s$ was demonstrated by mixing isolated $S c$ spores with a liquid culture of $B S$ followed by inoculation onto an agar swarm plate and incubation. After 5 days, $S c$ colonies are visible on the plate and are only dispersed in the presence of $B s$ in all samples tested $(n=13)$ (Fig. 1A). Spore dispersal by $B s$ occurs across the entire surface of the plate and to the plate's edge $(4.5 \mathrm{~cm}$ from the inoculation point). To demonstrate that the $S c$ spores are being moved by $B s$ cells and not merely "floating" in the expanding $B s$ colony, we conducted identical assays but inoculated the $S c$ spores and $B s$ culture separately and onto different areas of the plate. The resulting $S c$ colonies form streaks across the plate that emanate from the $B s$ inoculation site in a predictable manner in all samples tested $(n=10)$ (Fig. 1B). This experiment was repeated on $12 \mathrm{~cm}$ plates and demonstrate that $S c$ spores are dispersed to the edge of the plate, which is $10 \mathrm{~cm}$ from the spore innoculation point $(n=3)$ (Fig. S1A). To determine the effeciency of transport, this experiment was conducted with dilutions of $S c$ spores so that the total number of $S c$ colonies would germinate without overlap and could be individually counted. The results demonstrate that $86 \pm 5.6 \%$ of the apparent colonies are located outside of the initial innoculation point under these conditions $(n=4)$ (Fig. S1B).
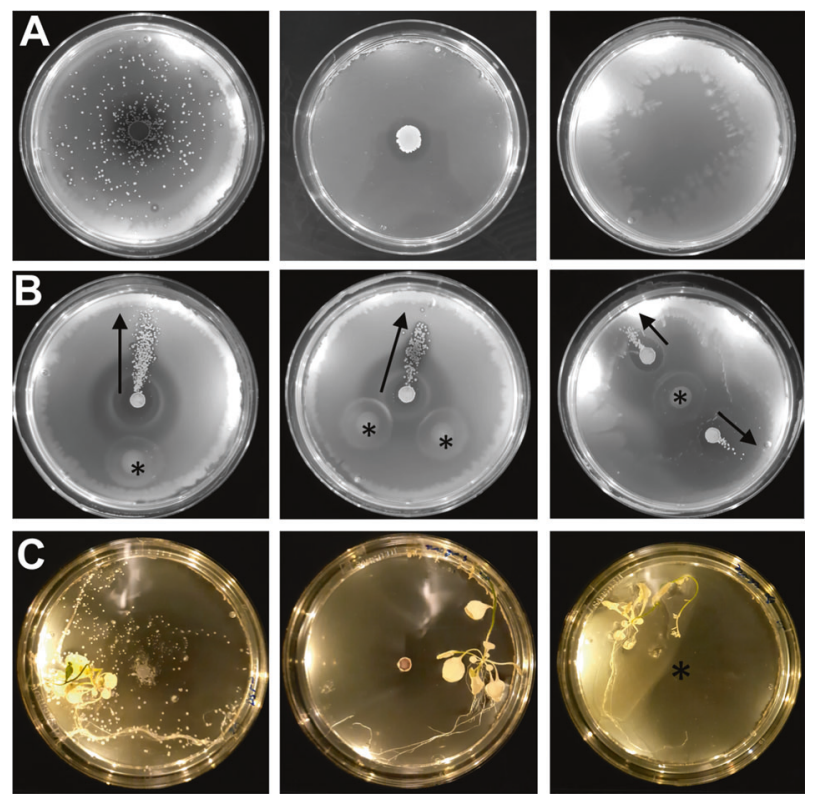

Fig. 1 S. coelicolor spores are transported by B. subtilis. A When $S c$ and $B s$ are innoculated on the center of a swarm plate, visible $S c$ colonies (white dots) are apparent and are only dispersed in the presence of motile $B s$. Left: $S c$ with $B s$. Middle: $S c$ alone. Right: $B s$ alone. B When $S c$ and $B s$ are innoculated in different positions on swarm plates, the $S c$ colonies are dispersed in the swarming direction of the $B s$ cells (black arrows). Asterisks denote the $B s$ innoculation sites. C $B S$ moves spores toward plant tissues. Left: $S c$ with $B s$. Middle: $S c$ alone. Right: $B s$ alone, asterisk denotes the $B s$ innoculation site.

\section{B. subtilis transports S. coelicolor spores to plant tissues}

In nature, $S c$ and $B s$ thrive near plant roots that excrete exudates but only $B s$ can move toward the root systems. We conducted assays to determine if $B s$ can transport $S c$ spores to plant tissues. Assays with the $B s$ strain alone demonstrate that the plates become "cloudy" with $B s$ cells in areas around plant tissues, perhaps due to the presence of nutritive plant exudates that facilitates bacterial growth (Fig. 1C). Like previous experiments, the $S c$ spores alone do not exhibit movement unless they are co-inoculated with $B S$ cells, and the dispersed spores preferentially establish colonies near plant tissues in all instances $(n=5)$ (Fig. 1C).

\section{Spore dispersal occurs with swarming and sliding Bs strains}

$B s$ has two modes of flagellar-mediated motility, swimming and swarming, that occur in liquid environments and on solid surfaces, respectively. When $B s$ senses that it is on a solid surface, it will differentiate into a swarmer cell that has a significant increase in the number of flagella and produces hydrophobic surfactants, such as surfactin, to efficiently spread across the surface [20-22]. In our experiments, we 


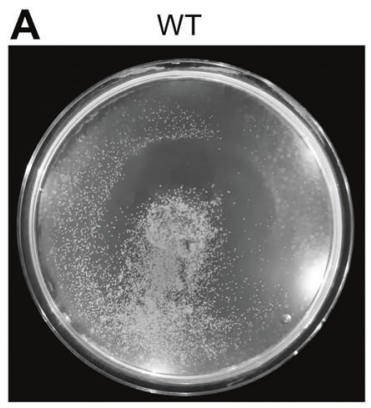

B

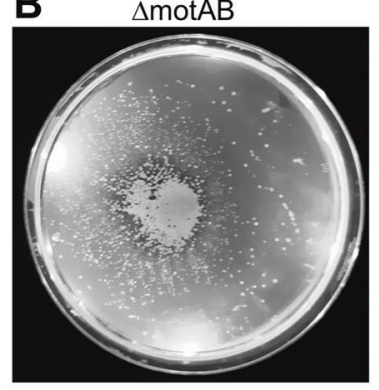

Lab-cultivated (LC)
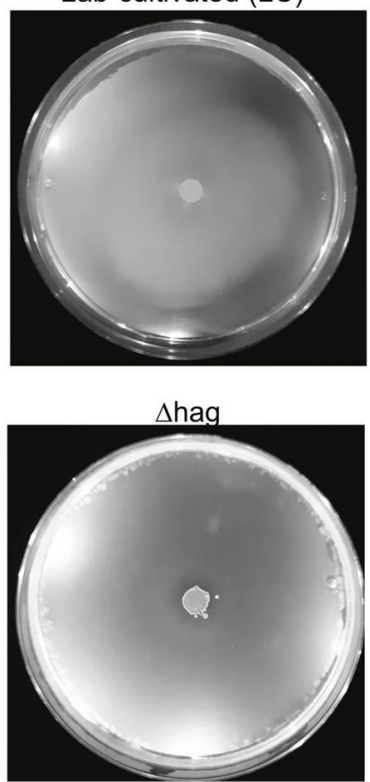

Fig. 2 B. subtilis can transport spores via swarming and sliding. A The WT (swarming strain) can transport spores on agar plates $(0.27-0.5 \%)(n=13)$, but swimming only strains (laboratory-cultivated WT strain or $\Delta$ epsH srfAA) cannot $(0.25-0.3 \%$ agar) $(n=6$ and $n=8$, respectively). Quantification of the results is shown in Fig. S2. B A $B s \Delta$ motAB strain that possesses flagella but lacks flagellar motility can disperse spores via sliding at WT levels $(n=6)$. A $B s$

utilized an undomesticated strain of Bs (NCIB3610) that can swarm, unlike common laboratory strains that lack the ability to differentiate into swarmer cells and fail to produce surfactin to undergo swarming motility (Table S1) [20, 22-24]. To determine if the $S c$ spores are transported by both swarming and swimming motilities, we repeated the experiments with a laboratory-cultivated $B s$ strain that is incapable of swarming, and spore transport does not occur on the agar swimming plates $(0.27 \%$ agar) (Figs. $2 \mathrm{~A}$ and S2) [20]. Importantly, the laboratory-cultivated strain has accumulated many genetic defects in addition to defective swarming capabilities. To ensure that the decrease in spore dispersal can be attributed to limitations of bacterial swimming, we utilized a $B s$ strain with an undomesticated genetic background but has been genetically altered so that it is only capable of swimming motilities ( $\triangle$ epsh srfAA, DK1484). Like the laboratory-cultivated strain, this strain does not disperse $S c$ spores on swim plates (Figs. 2A and S2). Therefore, we conclude that spore transport can be accomplished by swarming motility but not swimming motility.

In addition to flagellar motilities, $B s$ can also move through sliding on surfaces. To determine if spores can be dispersed through sliding, we utilized a $B s$ mutant that has flagella but the flagella cannot rotate $(\triangle \operatorname{mot} A B$, DS222) [25], and a mutant that does not possess flagella ( $\Delta$ hag, DS1677) [26]. The $\triangle$ mot $A B$ strain moves spores over the

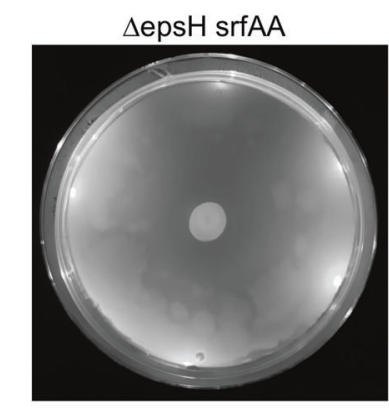

C

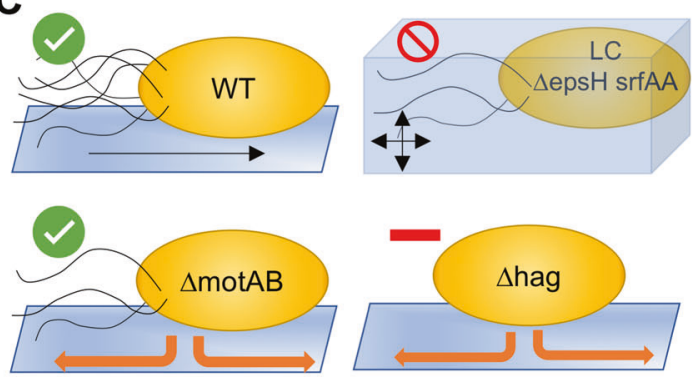

$\Delta$ hag strain that does not possess flagella cannot disperse the spores via sliding at WT levels $(n=6)$. Quantification of results is shown in Fig. S2. All $\Delta$ hag plates are shown in Fig. S3. C In summation, spores are dispersed by swarming (WT) and sliding $(\Delta \operatorname{mot} \mathrm{AB}) B s$ cells in a flagella-dependent manner. However, spores are not dispersed via swimming (lab-cultivated or $\Delta$ epsH srfAA), and are significantly less dispersed via sliding in the absence of flagella ( $\Delta$ hag).

entire surface and to the edge of the agar plates in all samples $(4.5 \mathrm{~cm}$ from the inoculation point) $(n=6)$ (Figs. 2B and S2). The number of dispersed spores cannot be ascertained due to overlapping colonies that result in apparent smears across the plates, but each plate contains over 100 dispersed $S c$ colonies. The $\Delta$ hag strain shows severely reduced dispersal, where an average of 3.2 spores are transported with an average maximum distance of $0.95 \pm 0.85 \mathrm{~cm}$ from the inoculation point $(n=6)$ (Figs. 2B, S2 and S3). Therefore, we surmise that spore dispersal can also occur through sliding but is facilitated by the presence of flagella (Fig. 2C). As an additional control, we also conducted these experiments by first spreading the $B s$ cells across the surface of the plate and then inoculating the $S c$ spores in the center. Spore dispersal was significantly reduced for both the $B s$ WT $(2.06 \pm 0.05 \mathrm{~cm})$ and $\Delta m o t A B$ $(1.25 \pm 0.16 \mathrm{~cm})$ strains when compared to co-inoculation in the center of the plate (Figs. S2 and S4). These data indicate that the $S c$ spores are transported by $B s$ cells that continuously move away from the inoculation point and are not transported after the motile $B s$ has covered the surface of the plate.

\section{S. coelicolor spores attach to $B$. subtilis flagella}

We utilized several microscopy methods to elucidate a mechanism for $S c$ spore dispersal by $B s$. Fluorescently 

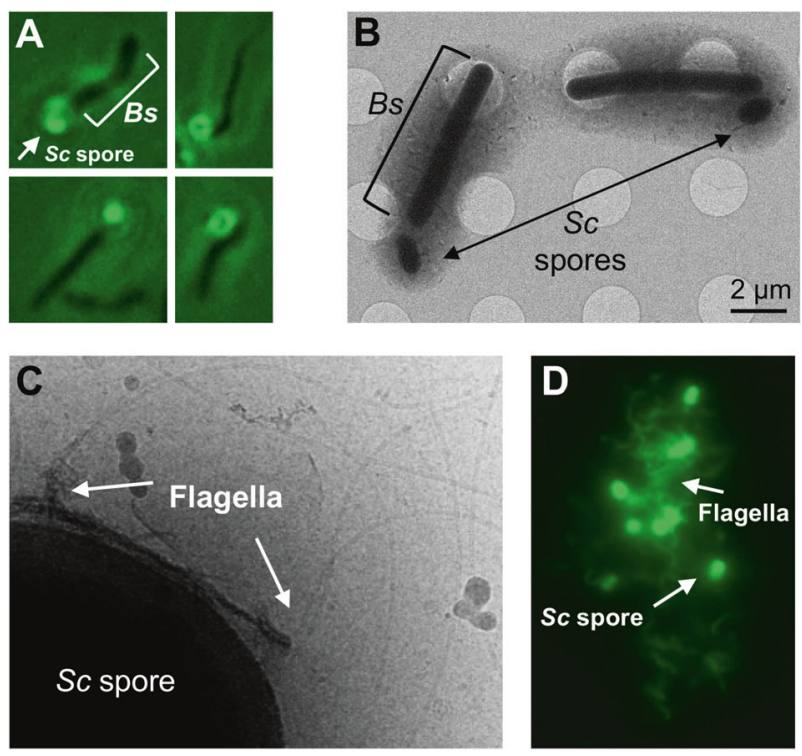

Fig. 3 Microscopy methods indicate that $S c$ spores directly adhere to $\boldsymbol{B} \boldsymbol{s}$ flagella. A Fluorescence microscopy of dye-labeled spores with unlabeled $B s$ cells demonstrate that the spores localize to the cell poles of Bs. B Cryo-electron microscopy samples of mixed $S c$ spores and $B s$ cells reveal that the spores do not directly adhere to the $B s$ cell body. C Cryo-EM shows the $B s$ flagella colocalize with the $S c$ spore coat. D Fluorescence microscopy of dye-labeled sheared flagella and spores demonstrate that spores directly interact with $B s$ flagella to form extended associations of both components.

labeled $S c$ spores were imaged under a fluorescence microscope and were immotile as expected (Supplementary Movie 1). However, when $B s$ cells were added to the fluorescent spores, the spores localize near the $B s$ cell poles (Supplementary Movie 2 and Fig. 3A). In some instances, the spores are stationary on the surface of the glass slide and an associated $B s$ cell is seen rotating around the spore (Movie 1). The observed $B s$ cell rotation in these assays is reminiscent of rotations seen in $B s$ cells that have their flagella chemically tethered to a solid surface, whereby the torque generated by the immobilized flagella induces rotation of the cell body [27]. This observation suggests that the $S c$ spores adhere directly to the $B s$ flagella, and therefore effectively mimic a flagellar tether in these instances. To verify that the $S c$ spores do not directly interact with the $B s$ cell body, we imaged a mixture of $S c$ spores and $B s$ cells with a cryo-electron microscope. Like the fluorescence microscopy images, the $S c$ spores are localized near the $B S$ cell poles but do not make direct contact with the $B s$ cell body (Figs. 3B and S5A). In total, $\sim 77 \%$ of the spores are located within $1 \mu \mathrm{m}$ of a $B s$ cell poll ( $n=35$ spores). To determine if the spores interact with $B s$ flagella, we utilized a $B s$ "minicell" strain ( $\min D:: T n Y L B)$ that lacks the excreted material inhibiting their direct visualization. Indeed, when mixed with $S c$ spores, the flagella can be seen co-localizing with the spores in two-dimensional cryoEM images (Figs. 3C and S5B).

$S c$ spore adherence to $B s$ flagella was further confirmed by visualization of a mixture of $S c$ spores with sheared flagella via fluorescence microscopy. Fluorescent labeling of $B s$ flagella was accomplished using a $B s$ strain (DS1919) that is mutated in a single flagellin residue (T209C) [28]. The surface-exposed thiol allows for direct labeling with dyes that possess a reactive maleimide group. This dye also labels proteins on the surface of the $S c$ spore coat. Indeed, when sheared $B s$ flagella are isolated and mixed with spores, and unassociated flagella are washed from the mixture, a majority of spores still retain associated flagella. In total, $\sim 64 \%$ of the spores are associated with flagella ( $n=130$ spores). In some instances, large "clumps" of spores entangled in flagella are observed (Figs. 3D and S5C). In comparison, the flagella without spores added do not form aggregates and are randomly dispersed (Fig. S5C).

\section{Hitchhiking is conserved in Streptomycetes}

To determine if spore dispersal by $B s$ also occurs in other Streptomyces species, we conducted $B s$ swarm plate assays with Streptomyces tendae (St), Streptomyces griseus $(\mathrm{Sg})$, Streptomyces scabies (Ss), and Streptomyces avermitilis $(\mathrm{Sa})$. To quantify spore dispersal, we prepared swarm plate assays where $B s$ cells were inoculated at the center of the plate $(9 \mathrm{~cm}$ in diameter) and the isolated spores are inoculated in four equidistant positions around the $B s$ inoculation site. The maximum dispersal distance, which is the distance from the center of the spore inoculation site to the most distant dispersed colony, was measured for each of the four spore samples. The wild-type (WT) $S c$ spores are dispersed by $B s$ in $100 \%$ of samples and are moved an average maximum distance of $2.67 \pm 0.43 \mathrm{~cm}$ from the initial inoculation point $(n=20)$. Likewise, these assays demonstrate that $S t(n=12), S g(n=8)$, and $S s(n=8)$ spores are dispersed in $100 \%$ of samples to similar distances as WT $S c$ spores. However, the $S a$ spores are dispersed at significantly shorter distances $(n=12)$ (Fig. 4A, B) and are dispersed in $83 \%$ of the samples. As the last common ancestor of $S c$ and $\mathrm{Sg}$ existed more than 200 million years ago and both species are capable of hitchhiking, these data suggest that the ancestor also possessed this dispersal mechanism and it remained conserved.

\section{Spore dispersal by $B$. subtilis is facilitated by the rodlin proteins}

The outer surface of most Streptomyces spores is characterized by a fibrillar rodlet layer, which is a striated pattern of pairwise aligned rodlets composed of the rodlin proteins [18, 19]. Scanning electron microscopy (SEM) 

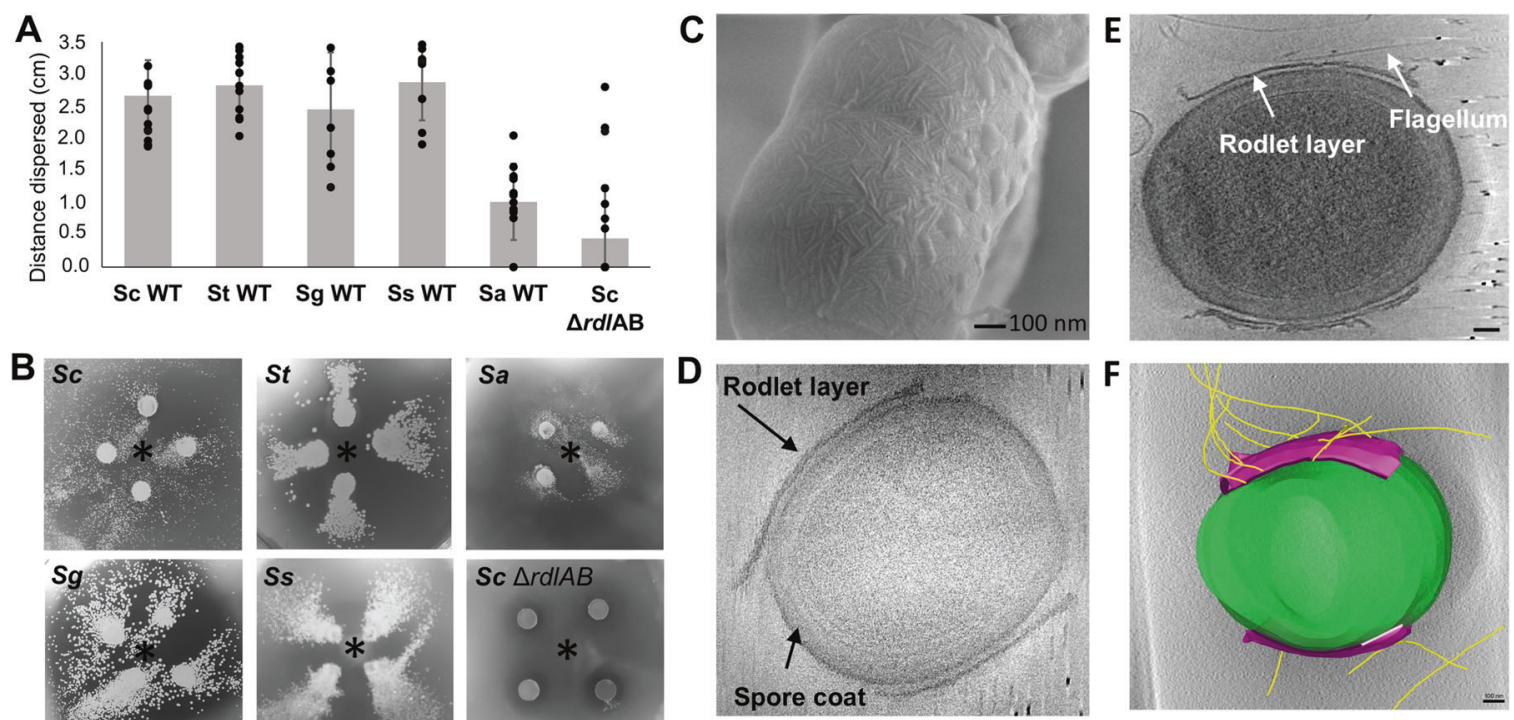

Fig. 4 Hitchhiking of Streptomyces spores is facilitated by the presence of rodlin proteins. A $B s$ swarm assays with both wild-type (WT) Streptomyces spores ( $S$. coelicolor $n=20, S$. tendae $n=12$, $S$. griseus $n=8, S$. scabies $n=8, S$. avermitilis $n=12$ ) and $S c$ spores lacking the rodlin proteins (Sc $\Delta r d l \mathrm{AB} n=24$ ) demonstrate that spores are dispersed in all tested WT species but with $S$. avermitilis dispersed the shortest distance, and dispersal is abrogated by the loss of the rodlins in Sc. Results are expressed as the mean of dispersal distance \pm standard error of the mean. Differences for $S c \Delta r d l \mathrm{AB}$ and $S a$ dispersal compared to WT $S c, S t, S g$, and $S s$ strains are statistically significant using a two-tailed null hypothesis significance test

$(p<0.05)$. B Representative images of $B s /$ spore swarm plates from A. The $B s$ innoculation site is denoted with an asterisk. C SEM of WT $S c$ spores shows the rodlet layer with pairwise rodlets of $20 \mathrm{~nm}$ spacing. D A representative cryo-ET image of isolated $S c$ WT spores shows that the rodlet layer does not cover the entire spore but leaves the poles exposed $(n=22)$. E Cryo-ET reconstructions show that flagella preferentially interact with the rodlet layer $(n=12)$. Scale bar $100 \mathrm{~nm}$. F Segmentation of the reconstruction from $\mathbf{E}$ clearly demonstrates the flagella:rodlin interaction. Spore body: green, rodlet layer: purple, flagella: yellow. Scale bar $100 \mathrm{~nm}$ (Color figure online).

images of $S c$ and $S s$ spores show the striated rodlet layer (Fig. 4C). In previous studies, the rodlets of Sc, S. lividans, $S t, S g$, and $S s$ were visually indistinguishable [19, 29]. Using electron microscopy images from this and previous studies, we measured the spacing of the rodlets in these species, which is highly conserved and around $\sim 20 \mathrm{~nm}$ (when measured from the center of the rodlet fibers) (Table S2). Furthermore, the rodlin proteins from $S c, S t$, and $S g$ have $\sim 34 \%$ sequence identity despite the species' distant evolutionary relation (Fig. S6) [19].

Intriguingly, $S a$ spores are less widely dispersed than the other Streptomyces species and it is the only tested species that natively lacks rodlin proteins [19]. In agreement, an $S c$ mutant strain that lacks the rodlin proteins $(\triangle r d l A B)$ abrogates hitchhiking by $B s(n=3$, Figs. S2 and S7). Importantly, previous studies demonstrate that the $S c \Delta r d l A B$ strain is not delayed in germination, and does not exhibit any behavioral or phenotypic change compared to the WT strain with the exception of the rodlet layer $[18,19,30]$. In contrast, Sc mutants that lack proteins which produce polysaccharides found on the surface of Streptomycetes $(\triangle c s l A$ and $\triangle m a t A B)$ are unaffected $(n=3$, Figs. S2 and S7) [31]. However, spore dispersal was not completely abolished in the $\triangle r d l A B$ strain. Using identical swarm plate assays described in the section above, the $\triangle r d l A B$ strain is

dispersed with an average maximum distance of $0.46 \pm$ $0.82 \mathrm{~cm}$ from the initial inoculation point and dispersal occurs in $33 \%$ of the samples $(n=24)$ (Fig. 2).

To characterize how rodlins interact with flagella in three dimensions, we conducted cryo-ET experiments of samples containing $B s$ minicells and $S c$ spores. Reconstructions show that the $S c$ spores are oval shaped and possess a thick coat. The rodlet layer can be seen as a sheath around the lateral sides of the spore with frayed edges, leaving the poles exposed, and suggest that the rodlet sheath easily peels away from the cell body $(n=22)$ (Fig. 4D). A similar spore morphology has also been observed in Streptomyces albus [32]. Bs flagella accumulate around and directly interact with the rodlet layer $(n=12)$ (Fig. $4 \mathrm{E}, \mathrm{F}$ and Movie 2). However, due to the thickness of the spores the resolution is limited and we could not deduce if the flagella preferentially bind specific features of the rodlet layer. Collectively, these data suggest that the rodlet layer facilitates spore dispersal by interacting directly with flagella.

\section{Hitchhiking of S. coelicolor spores is not limited to Bacillus}

Although B. subtilis is ubiquitous in soil, other genera are also flagellated and may also contribute to dispersal of $S c$ 


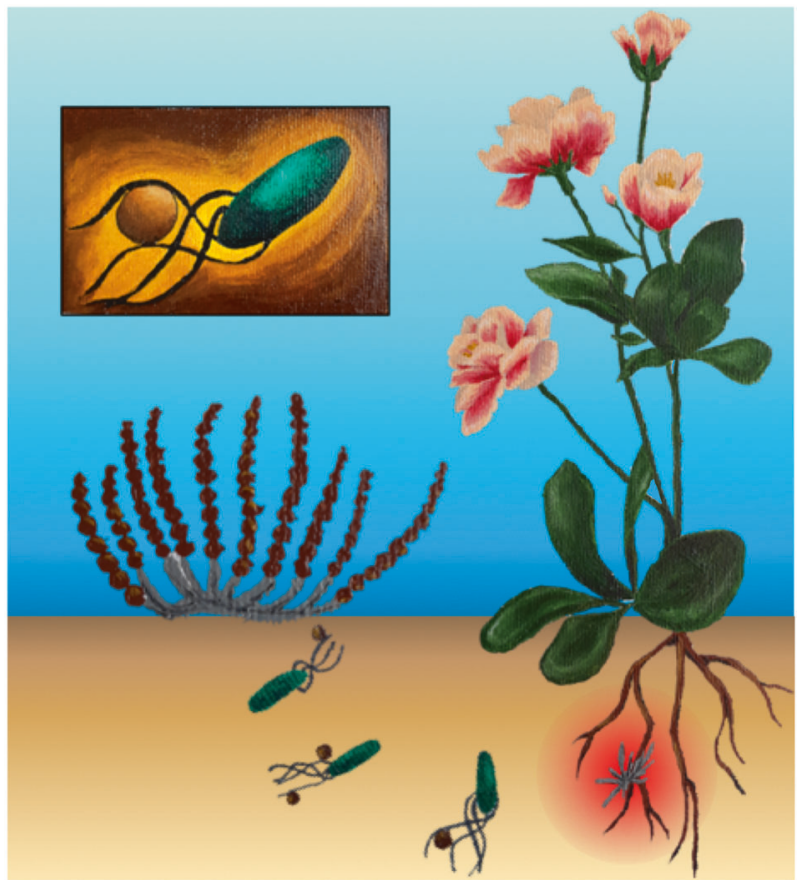

Fig. 5 An overview of the hitchhiking model. Aerial Streptomyces spores are transported on the $\mathrm{cm}$-scale to plant root systems by directly adhering to the flagella of motile bacteria (inset). Here, the spores germinate and produce antibiotics (red gradient) to ward off microbial competitors (Color figure online).

spores. We therefore conducted swarm plate assays with a $P$. fluorescens WT strain (R1SS101), which is also natively associated with plant roots [33]. Importantly, $P f$ disperses spore similarly to $B s$, but $S c$ colonies appear in patterns that are reminiscent of Pseudomonas swarm patterns [34] $(n=6)$ (Fig. S8). These data demonstrate that hitchhiking is a widespread mechanism that allows Streptomyces spores to disperse at cm-scales (Fig. 5).

\section{Discussion}

Sessile Streptomycetes have a complex lifecycle that involves formation of aerial hyphae that differentiate into spores. The spores of some Streptomyces species, including $S c$, are dispersed over long distances by direct attachment to insects and nematodes [6]. Intriguingly, recent reports identify that specific volatile metabolites secreted by Streptomycetes attract arthropods as a mechanism for spore dispersal [7], and they can induce the formation of Streptomyces "explorer cells", while simultaneously starving microbial competitors [35]. However, it is unclear how the spores are dispersed specifically at the centimeter scale to plant root microenvironments. Here, we demonstrate that Streptomyces spores are able to utilize the motility machinery of motile soil microbes by directly attaching to their flagella. While these experiments demonstrate that $S c$ spores are dispersed by $B s$ and $P f$ regardless of their destination, these motile bacteria are also known to associate with plant roots. Therefore, this mechanism of dispersal, called hitchhiking, may provide Streptomyces spores a mechanism for translocation to beneficial environments. Indeed, assays with $A$. thaliana plants demonstrate that $B S$ can transport $S c$ spores to plant tissues. This allows spores to germinate near nutrient-rich plant exudate to generate filamentous colonies that produce antibiotics, thereby protecting the plant from potential phytopathogens.

Hitchhiking is facilitated by two spore coat proteins, RdlA and RdlB, which are conserved in most Streptomycetes. These proteins assemble into pairwise aligned filaments, called rodlets, on the outer surface of the spores and are spaced $\sim 20 \mathrm{~nm}$ apart (Table S2). Until now, the function of the rodlets has remained elusive [30]. Interestingly, the diameter of the bacterial flagellar filament is also $\sim 20 \mathrm{~nm}$ $[22,36]$. Therefore, it is possible that the rodlet layer provides a gripped surface for the flagella, which become "wrapped" in the grooves made by the rodlin proteins and thereby facilitates spore transport. However, our cryo-ET data can not support such speculations due to the limited resolution of the whole-cell reconstructions. Therefore, it is still unclear what properties of the rodlet layer encourage interactions with flagella.

Emerging studies have demonstrated that flagella preferentially interact with hydrophobic surfaces and flagellin can undergo methylation to increase flagella hydrophobicity [37-39]. This increase in hydrophobicity allows pathogenic bacteria to adhere to host cells [37-39], and flagellar adherence to plant cells has also been implicated in establishing colonization [40, 41]. Flagella hydrophobicity may facilitate interactions with hydrophobic spores and may account for spore transport that is seen in the absence of rodlins, given that the spore surface without rodlins remains hydrophobic (in $S a$ WT and $S c$ $\Delta r d l \mathrm{AB}$ strains) $[18,42,43]$. Therefore, the same flagellar interactions that facilitate adherence to plant roots may also contribute to adherence to spores, and necessitate that motile bacteria not evolve to eliminate this interaction. Since spore:flagella interactions are in-part facilitated by hydrophobic interactions, this mode of transport may be influenced by environmental factors that impact Van der Waals screening distances such as salt concentrations and $\mathrm{pH}$.

Although spore hitchhiking seems disadvantageous to the motile partner, previous research has shown that fungal hyphae can form so-called "fungal highways" that serve as bridges for motile bacteria over air gaps (such as air gaps found in soil) [14, 44, 45]. Like fungi, Steptomycetes form 
aerial hyphae that are structurally similar to fungal hyphae [46]. Therefore, Streptomycetes may be able to form "bacterial bridges" for their motile partner, but such interactions have not been reported yet. If such "bacterial bridges" do form, this would supply the system with a synergistic transport that has been previously observed between nonmotile fungal spores and motile bacteria [14].

The hitchhiking model is supported by a previous study that examines the interaction of two other root-colonizing microbes: the immotile fungus $A f$ and the motile bacterium Paenibacillus vortex $(P v)$ [14]. Af spores are demonstrated to be dispersed by $P v$ in a swarming-dependent manner via direct attachment to flagella; dispersal is abrogated by the addition of excess purified $P v$ flagella or perturbations to the $A f$ spore coat [14]. Furthermore, scanning EM micrographs show direct contact between $P v$ flagella and $A f$ spores [14]. Although this study does not identify the spore coat component(s) responsible for adherence to flagella, Aspergillus spores also possess a rodlet layer [47]. Additionally, this study demonstrates that some Penicillium species are also transported by $P v$, and these fungi possess a rodlet layer [48]. Collectively, these data may suggest that hitchhiking of spores onto motile bacteria via the formation of a striated rodlet layer is a dispersal mechanism that convergently evolved in both domains of life.

The colonization of plant roots by some Streptomycetes improves plant health and performance in a natural and sustainable manner [2-5]. Therefore, our data are applicable to industrial initiatives that aim to improve soil conditions for Streptomyces root colonization. Likewise, many Aspergillus fungi, like $A f$ and $A$. niger, are human and plant pathogens. Therefore, insights into hitchhiking of these sessile organisms may elucidate unknown infection mechanisms.

\section{Methods and materials}

\section{Streptomyces spore isolation}

The following Streptomyces strains were used in this study: S. coelicolor M145 [49], S. coelicolor $\triangle$ rdlAB6 [42], S. tendae Tü901/8c [50], S. griseus (ATCC 13273), S. avermitilis (ATCC 31267), and S. scabies ISP5078. Spores were harvested from MS agar plates and quantified as described before [49].

\section{Bacillus subtilis cultivation}

The undomesticated Bs strain NCIB3610 [23] 25\% glycerol stock was placed into $5 \mathrm{ml}$ of LB and grown overnight at $30^{\circ} \mathrm{C}$. After $16 \mathrm{~h}$ of growth, $100 \mu \mathrm{l}$ of the overnight culture was diluted into $5 \mathrm{ml}$ of LB and grown at $37{ }^{\circ} \mathrm{C}$ to an O.D. of $0.4-0.5$.

\section{Pseudomonas fluorescens cultivation}

The $P f$ strain R1SS101 25\% glycerol stock was placed into $5 \mathrm{ml}$ of $50 \% \mathrm{~TB}$ and grown overnight at $30^{\circ} \mathrm{C}$. After $16 \mathrm{~h}$ of growth, $100 \mu \mathrm{l}$ of the overnight culture was diluted into $5 \mathrm{ml}$ of $50 \% \mathrm{~TB}$ and grown at $37^{\circ} \mathrm{C}$ to an O.D. of 0.4-0.5.

\section{Swarm and swim plate assays}

Swarm and swim plates were conducted on nutrient broth plates $(0.5 \%$ peptone, $0.3 \%$ yeast extract, $0.5 \% \mathrm{NaCl})$ containing specific amounts of agar (0.27-0.5\%). All components were mixed, autoclaved for $20 \mathrm{~min}$, and $30 \mathrm{ml}$ of the media was poured into a plastic petri dish with a $9 \mathrm{~cm}$ diameter. The plates were cooled for $30 \mathrm{~min}$ in a sterile fume hood and then stored in a $4{ }^{\circ} \mathrm{C}$ fridge for a maximum of 1 week. To determine if $S c$ spores are dispersed by $B S$, $3 \mu \mathrm{l}$ of $B s$ cells are inoculated onto the plate and $3 \mu \mathrm{l}$ of $S c$ spore stocks are either added on the $B s$ inoculation site or to a separate inoculation site. The plates are incubated at $30^{\circ} \mathrm{C}$ for 5 days and imaged on a light box.

For plates that first had $B s$ cells spread across the surface, $100 \mu \mathrm{l}$ of $B s$ cells at an O.D. 0.4-0.5 were pipetted onto the plate $(0.5 \%$ agar $)$ and then spread over the surface of the plate using an L-spreader. Then $3 \mu \mathrm{l}$ of $S c$ spore stock was added to the center of the plate. Plates were incubated at $30{ }^{\circ} \mathrm{C}$ for 5 days and imaged on a light box.

For all plate images, the distance of spore dispersal was determined using Image $\mathbf{J}$ bundled with Java 1.8.0_172 software. To measure the distances, the diameter of the petri dish $(9 \mathrm{~cm})$ was used as a reference scale. Then, a line was drawn from the inoculation point of the spores to the Streptomyces colony that is furthest from the inoculation point. The distance of the line was calculated based on the value of the reference scale.

\section{Fluorescence microscopy}

For fluorescent labeling of spores with unlabeled B. subtilis, $10 \mu \mathrm{l}$ of $S c$ spore stock was added to $1 \mathrm{ml}$ of iced LB. The $1 \mu \mathrm{l}$ of the fluorescent styryl dye, FM2-10 (Thermo Fisher Scientific), was added to the $1 \mathrm{ml}$ solution and inverted to mix. Excess dye was removed by rinsing the spores $4 \mathrm{X}$ with $1 \mathrm{ml}$ of iced LB via centrifugation and decanting. After the final decantation, $1 \mathrm{ml}$ of $B s$ cells with an O.D. of 0.4 were added to the spores, mixed via pipetting, and incubated at ambient temperatures for $5 \mathrm{~min}$. Immediately before imaging, $5 \mu \mathrm{l}$ of the samples were placed on a glass slide and a glass coverslip was placed on top. The sample was imaged on a Zeiss Axioscope A1 fluorescent microscope scope equipped with an Axiocam Mrc5 camera (Zeiss) in the Institute of Biology Microscopy Unit using a GFP filter. Images were collected and processed using Axiovision software (Zeiss). 
For fluorescent labeling of sheared B. subtilis flagella with spores, $B$. subtilis (strain $\Delta$ hag amyE::PhaghagT209C spec) was grown to an O.D. of 0.6, and $2 \mathrm{ml}$ of the cells were pelleted via centrifugation and resuspended in $1 \mathrm{ml}$ of PBS buffer $\mathrm{pH} 7.5$. The $1 \mathrm{ml}$ suspension was passed back-and-forth between two $5 \mathrm{ml}$ syringes with 21 gauge needles that were connected by a plastic tube $(10 \mathrm{~cm}$ long with an inner diameter of $0.58 \mathrm{~mm})$. The cell suspension was gently passed back and forth between the syringes 50 times, with $1 \mathrm{~min}$ pauses every ten passes. The cells were removed from the mixture via centrifugation at $5000 \times g$ for $5 \mathrm{~min}$. The supernatant containing the flagella were then centrifuged once again to remove any residual cells. In total, $5 \mu \mathrm{g} / \mathrm{ml}$ Alexa Fluor $488 \mathrm{C}_{5}$ maleimide dye was added to the suspension and incubated for $5 \mathrm{~min}$ at room temperature. In total, $10 \mu \mathrm{l}$ of Sc WT spore stock was then added to the mixture. The tube was gently inverted 50 times and the spores with associated flagella were pelleted via centrifugation at $5000 \times g$ for $5 \mathrm{~min}$. The pellet was washed with $1 \mathrm{ml} \mathrm{PBS}$ buffer $\mathrm{pH} 7.5$ and centrifuged once again. The resulting pellet was resuspended in $50 \mu \mathrm{PBS}$ buffer $\mathrm{pH}$ 7.5. The sample was imaged on a Zeiss Axioscope A1 fluorescent microscope scope equipped with an Axiocam Mrc5 camera (Zeiss) in the Institute of Biology Microscopy Unit using a GFP filter. Images were collected and processed using Axiovision software (Zeiss).

\section{Cryo-electron microscopy}

B. subtilis cells were grown to an O.D. of 0.5 and $1 \mathrm{ml}$ of $B$. subtilis cells were mixed with $5 \mu \mathrm{l} S$. coelicolor spores glycerol stock and incubated at ambient temperatures for $5 \mathrm{~min}$. Cells were concentrated by centrifugation and $3 \mu \mathrm{l}$ aliquots of the cell suspension are applied to glowdischarged R2/2 200 mesh copper Quantifoil grids (Quantifoil Micro Tools), the sample was pre-blotted for $30 \mathrm{~s}$, and then blotted for $2 \mathrm{~s}$. Grids were pre-blotted and blotted at $20{ }^{\circ} \mathrm{C}$ and at $95 \%$ humidity. The grids were plunge frozen in liquid ethane using an automated Leica EM GP system (Leica Microsystems) and stored in liquid nitrogen. The grids were imaged on a $120 \mathrm{kV}$ Talos L120C cryo-electron microscope (Thermo Fisher Scientific) at the Netherlands Center for Electron Nanoscopy.

\section{Cryo-electron tomography}

B. subtilis mini-cell strain was grown from a $20 \%$ glycerol stock to an O.D. of 0.6 in $50 \mathrm{ml}$ of LB. The cells were centrifuged at $8000 \times g$ for $30 \mathrm{~min}$. The supernatant was collected and then centrifuged at $12,000 \times g$ for $20 \mathrm{~min}$. The resulting cell pellet was resuspended in $20 \mu \mathrm{l}$ of LB and $8 \mu \mathrm{l}$ of WT $S c$ spore stock was added to the cell mixture. A 1/10 dilution of protein A- treated 10-nm colloidal gold solution (Cell Microscopy Core, Utrecht University, Utrecht, The Netherlands) was added to the mixture and mixed by pipetting. The grids were prepared using an automated Leica EM GP system (Leica Microsystems) with the sample chamber set at $20^{\circ} \mathrm{C}$ and at $95 \%$ humidity. In total, $3 \mu \mathrm{l}$ of the sample mixture was applied to a freshly glow-discharged copper R2/2 200 grid (Quantifoil Micro Tools), pre-blotted for $30 \mathrm{~s}$, and then blotted for $2 \mathrm{~s}$. The grid was plunge frozen in liquid ethane and stored in liquid nitrogen.

Images were recorded with a Gatan K3 Summit direct electron detector equipped with a Gatan GIF Quantum energy filter with a slit width of $20 \mathrm{eV}$. Images were taken at a magnification of $\times 19,500$, which corresponds to a pixel size of $4.4 \AA$. Tilt series were collected using SerialEM with a bidirectional dose-symmetric tilt scheme $\left(-60^{\circ}\right.$ to $60^{\circ}$, starting from $0^{\circ}$ ) with a $2^{\circ}$ increment. The defocus was set to $-12 \mu \mathrm{m}$ and the cumulative exposure per tilt series was $160 \mathrm{e}-/ \mathrm{A}^{2}$. Bead tracking-based tilt series alignment and drift correcting were done using IMOD [51] and CTFplotter was used for contrast transfer function determination and correction [52]. Tomograms were reconstructed using simultaneous iterative reconstruction with iteration set to 4 . Segmentation was done in IMOD.

\section{Plant growth}

Arabidopsis thaliana Col-0 strain was grown from sterilized seedlings on sterilized plant MS agar media. Harvested A. thaliana seeds were sterilized in a sterile fume hood by incubation in $10 \%$ bleach for $30 \mathrm{~min}$, washed with sterile water, and then incubated in $70 \%$ ethanol for $5 \mathrm{~min}$. The seeds were then washed $6 \mathrm{X}$ with sterile water, placed on sterile filter paper, and placed in a dark $4{ }^{\circ} \mathrm{C}$ fridge for 3-4 days in a sterile and parafilm-sealed petri dish. Plant agar media plates were prepared by autoclaving Murashige and Skoog (MS) media (0.22\% MS media with vitamins, $1.2 \%$ plant agar, $0.5 \%$ sucrose, $\mathrm{pH} 5.8$ ) and pouring $100 \mathrm{ml}$ of the media into square petri dishes with $12 \mathrm{~cm}$ length. The plates were allowed to cool for $1 \mathrm{~h}$ in a sterile fume hood. $A$. thaliana seeds were manually placed on the surface of the plates $1 \mathrm{~cm}$ apart by picking up the seeds with sterilized wooden picks. The plates were sealed with parafilm and placed in a climate-controlled plant growth chamber at a $20^{\circ}$ angle so the plant roots grew on the surface of the media. The plant chamber was kept at $21{ }^{\circ} \mathrm{C}$ with a $16-\mathrm{h}$ light cycle. The plants were allowed to grow for 1 month before use in chemoattraction assays (below).

\section{Chemotaxis attractant assays with plant roots}

Chemoattraction of $B s$ cells to plant roots in the presence and absence of $S c$ spores was conducted on minimal media 
plates with $0.25 \%$ agar. The media was prepared according to previous methods [53] in round petri dishes with $9 \mathrm{~cm}$ diameter. One month old sterile A. thaliana plants were removed from their sterile media and placed on the edge of the minimal media plates. In total, $3 \mu \mathrm{l}$ of $B$. subtilis culture was placed to the center of the plate, and then $3 \mu l$ of the isolated spore stock was also added to the center. Controls of each bacteria by itself were also prepared. The plates were incubated for $16 \mathrm{~h}$ at $30^{\circ} \mathrm{C}$ and then placed in a climate-controlled plant growth chamber for 2 weeks. The plant chamber was kept at $21^{\circ} \mathrm{C}$ with a 16-h light cycle. After $S c$ colonies were visible, the plates were imaged on a light box.

Acknowledgements We thank Dr. Daniel Kearns for his guidance and advice during the preparation of this manuscript. We also thank him for the following B. subtilis strains: DK605, DS1677, DS222, DS1919, and DK1484. Mark Ladinsky at Caltech for the 3D segmentation of the $S c$ spores with $B s$ minicells, Dr. Jos Raaijmakers for the P. fluorescens strain R1SS101 and the A. thaliana Col-0 strain, Dr. Chris Rao for the undomesticated B. subtilis strain NCIB3610, Dr. Rose Loria for the $S$. scabies ISP5078 strain, and Dr. Joost Willemse for assistance with the fluorescence microscopy experiments. We also thank Dr. Jos Raaijmakers and Dr. Gilles van Wezel for their on-going support and advice with this project. We thank the Netherlands Centre for Electron Nanoscopy (NeCEN) for access to cryo-EM data collection and processing facilities, and the Institute of Biology Microscopy Unit at Leiden University for access to and training with light and fluorescence microscopes. This work is part of the research program National Roadmap for Large-Scale Research Infrastructure 2017-2018 with project number 184.034.014, which is financed in part by the Dutch Research Council (NWO). This project was funded by the European Union under a Marie-Sklodowska-Curie COFUND LEaDing fellowship to ARM, and an NWO Talent Programme Veni grant to ARM.

Author contributions ARM, DC, and $\mathrm{AB}$ designed research; ARM and DC conducted experiments; ARM and DC analyzed data; ARM, $\mathrm{DC}$, and $\mathrm{AB}$ wrote the manuscript.

\section{Compliance with ethical standards}

Conflict of interest The authors declare no competing interests.

Publisher's note Springer Nature remains neutral with regard to jurisdictional claims in published maps and institutional affiliations.

Open Access This article is licensed under a Creative Commons Attribution 4.0 International License, which permits use, sharing, adaptation, distribution and reproduction in any medium or format, as long as you give appropriate credit to the original author(s) and the source, provide a link to the Creative Commons license, and indicate if changes were made. The images or other third party material in this article are included in the article's Creative Commons license, unless indicated otherwise in a credit line to the material. If material is not included in the article's Creative Commons license and your intended use is not permitted by statutory regulation or exceeds the permitted use, you will need to obtain permission directly from the copyright holder. To view a copy of this license, visit http://creativecommons. org/licenses/by/4.0/.

\section{References}

1. van der Meij A, Worsley SF, Hutchings MI, van Wezel GP. Chemical ecology of antibiotic production by actinomycetes. FEMS Microbiol Rev. 2017;41:392-416.

2. Worsley SF, Newitt J, Rassbach J, Batey SFD, Holmes NA, Murrell JC, et al. Streptomyces endophytes promote host health and enhance growth across plant species. Appl Environ Microbiol. 2020;86:1-35.

3. Vurukonda SSKP, Giovanardi D, Stefani E. Plant growth promoting and biocontrol activity of streptomyces spp. as endophytes. Int J Mol Sci. 2018;19:1-26.

4. Franco C, Michelsen P, Percy N, Conn V, Listiana E, Moll S, et al. Actinobacterial endophytes for improved crop performance. Australas Plant Pathol. 2007;36:524-31.

5. Olanrewaju OS, Babalola OO. Streptomyces: implications and interactions in plant growth promotion. Appl Microbiol Biotechnol. 2019;103:1179-88.

6. Ruddick SM, Williams ST. Studies on the ecology of actinomycetes in soil V. some factors influencing the dispersal and adsorption of spores in soil. Soil Biol Biochem. 1972;4:93-103.

7. Becher PG, Verschut V, Bibb MJ, Bush MJ, Molnár BP, Barane E, et al. Developmentally regulated volatiles geosmin and 2methylisoborneol attract a soil arthropod to Streptomyces bacteria promoting spore dispersal. Nat Microbiol. 2020;5:821-9.

8. Jarrell KF, McBride MJ. The surprisingly diverse ways that prokaryotes move. Nat Rev Microbiol. 2008;6:466-76.

9. Muok AR, Briegel A. Intermicrobial Hitchhiking: how nonmotile microbes leverage communal motility. Trends Microbiol. 2020:1-9.

10. Samad T, Billings N, Birjiniuk A, Crouzier T, Doyle PS, Ribbeck K. Swimming bacteria promote dispersal of non-motile staphylococcal species. ISME J. 2017;11:1933-7.

11. Xiong L, Cao Y, Cooper R, Rappel WJ, Hasty J, Tsimring L. Flower-like patterns in multi-species bacterial colonies. elife 2020;9:1-27.

12. Hagai E, Dvora R, Havkin-Blank T, Zelinger E, Porat Z, Schulz S, et al. Surface-motility induction, attraction and hitchhiking between bacterial species promote dispersal on solid surfaces. ISME J. 2014;8:1147-51.

13. Finkelshtein A, Roth D, Ben JE, Ingham CJ. Bacterial swarms recruit cargo bacteria to pave the way in toxic environments. mBio. 2015;6:1-10.

14. Inghama CJ, Kalismand O, Finkelshteind A, Ben-Jacob E. Mutually facilitated dispersal between the nonmotile fungus Aspergillus fumigatus and the swarming bacterium Paenibacillus vortex. Proc Natl Acad Sci USA. 2011;108:19731-6.

15. Shrivastava A, Patel VK, Tang Y, Yost SC, Dewhirst FE, Berg HC. Cargo transport shapes the spatial organization of a microbial community. Proc Natl Acad Sci USA. 2018;115:8633-8.

16. Rowbotham TJ. Preliminary report on the pathogenicity of Legionella pneumophila for freshwater and soil amoebae. J Clin Pathol. 1980;33:1179-83.

17. Allard-massicotte R, Tessier L, Lécuyer F, Lakshmanan V, Lucier J. Bacillus subtilis early colonization of Arabidopsis thaliana roots. mBio. 2016;7:1-10.

18. Yang W, Willemse J, Sawyer EB, Lou F, Gong W, Zhang H, et al. The propensity of the bacterial rodlin protein RdlB to form amyloid fibrils determines its function in Streptomyces coelicolor. Sci Rep. 2017;7:1-13.

19. Claessen D, Stokroos L, Deelstra HJ, Penninga NA, Bormann C, Salas JA, et al. The formation of the rodlet layer of streptomycetes is the result of the interplay between rodlins and chaplins. Mol Microbiol. 2004;53:433-43. 
20. Patrick JE, Kearns DB. Laboratory strains of Bacillus subtilis do not exhibit swarming motility. J Bacteriol. 2009;191:7129-33.

21. Najafi J, Shaebani MR, John T, Altegoer F, Bange G, Wagner C. Flagellar number governs bacterial spreading and transport efficiency. Sci Adv. 2018;4:1-9.

22. Mukherjee $S$, Kearns DB. The structure and regulation of flagella in Bacillus subtilis. Annu Rev Genet. 2014;48:319-40.

23. Nye TM, Schroeder JW, Kearns DB, Simmons LA. Complete genome sequence of undomesticated Bacillus subtilis strain NCIB 3610. Genome Announc. 2017;5:12-3.

24. Kearns DB, Losick R. Swarming motility in undomesticated Bacillus subtilis. Mol Microbiol. 2003;49:581-90.

25. Chan JM, Guttenplan SB, Kearns DB. Defects in the flagellar motor increase synthesis of poly- $\gamma$-glutamate in Bacillus subtilis. $\mathrm{J}$ Bacteriol. 2014;196:740-53.

26. Mukherjee S, Babitzke P, Kearns DB. FliW and flis function independently to control cytoplasmic flagellin levels in Bacillus subtilis. J Bacteriol. 2013;195:297-306.

27. Glekas GD, Cates JR, Cohen TM, Rao CV, Ordal GW. Sitespecific methylation in Bacillus subtilis chemotaxis: Effect of covalent modifications to the chemotaxis receptor McpB. Microbiology. 2011;157:56-65.

28. Guttenplan SB, Shaw S, Kearns DB. The cell biology of peritrichous flagella in Bacillus subtilis. Mol Microbiol. 2013;87:211-29.

29. Di Berardo C, Capstick DS, Bibb MJ, Findlay KC, Buttner MJ, Elliot MA. Function and redundancy of the chaplin cell surface proteins in aerial hypha formation, rodlet assembly, and viability in Streptomyces coelicolor. J Bacteriol. 2008;190:5879-89.

30. Elliot MA, Karoonuthaisiri N, Huang J, Bibb MJ, Cohen SN, Kao $\mathrm{CM}$, et al. The chaplins: a family of hydrophobic cell-surface proteins involved in aerial mycelium formation in Streptomyces coelicolor. Genes Dev. 2003;17:1727-40.

31. van Dissel D, Willemse J, Zacchetti B, Claessen D, Pier GB, van Wezel GP. Production of poly- $\beta-1,6-\mathrm{N}$-acetylglucosamine by MatAB is required for hyphal aggregation and hydrophilic surface adhesion by Streptomyces. Micro Cell. 2018;5:269-79.

32. Sexton DL, Tocheva EI. Ultrastructure of exospore formation in Streptomyces revealed by cryo-electron tomography. Front Microbiol. 2020;11:1-9.

33. Oku S, Komatsu A, Nakashimada Y, Tajima T, Kato J. Identification of Pseudomonas fluorescens chemotaxis sensory proteins for malate, succinate, and fumarate, and their involvement in root colonization. Microbes Environ. 2014;29:413-9.

34. Kollaran AM, Joge S, Kotian HS, Badal D, Prakash D, Mishra A, et al. Context-specific requirement of forty-four two-component loci in Pseudomonas aeruginosa swarming. iScience. 2019;13:305-17.

35. Jones SE, Pham CA, Zambri MP, McKillip J, Carlson EE, Elliot MA. Streptomyces volatile compounds influence exploration and microbial community dynamics by altering iron availability. mBio. 2019;10:1-18.

36. Imada K. Bacterial flagellar axial structure and its construction. Biophys Rev. 2018;10:559-70.
37. Friedlander RS, Vogel N, Aizenberg J. Role of flagella in adhesion of Escherichia coli to abiotic surfaces. Langmuir. 2015;31:6137-44.

38. Horstmann JA, Lunelli M, Cazzola H, Heidemann J, Kühne C, Steffen P, et al. Methylation of Salmonella Typhimurium flagella promotes bacterial adhesion and host cell invasion. Nat Commun. 2020;11:1-11.

39. Lillehoj EP, Kim BT, Kim KC. Identification of Pseudomonas aeruginosa flagellin as an adhesin for Muc1 mucin. Am J Physiol Lung Cell Mol Physiol. 2002;282:751-6.

40. Rossez Y, Wolfson EB, Holmes A, Gally DL, Holden NJ. Bacterial flagella: twist and stick, or dodge across the kingdoms. PLoS Pathog. 2015;11:1-15.

41. Wheatley RM, Poole PS. Mechanisms of bacterial attachment to roots. FEMS Microbiol Rev. 2018;42:448-61.

42. Claessen D, Wösten HAB, Van Keulen G, Faber OG, Alves AMCR, Meijer WG, et al. Two novel homologous proteins of Streptomyces coelicolor and Streptomyces lividans are involved in the formation of the rodlet layer and mediate attachment to a hydrophobic surface. Mol Microbiol. 2002;44:1483-92.

43. Claessen D, Rink R, De Jong W, Siebring J, De Vreugd P, Boersma FGH, et al. A novel class of secreted hydrophobic proteins is involved in aerial hyphae formation in Streptomyces coelicolor by forming amyloid-like fibrils. Genes Dev. 2003;17:1714-26.

44. Kohlmeier S, Smits THM, Ford RM, Keel C, Harms H, Wick LY. Taking the fungal highway: Mobilization of pollutant-degrading bacteria by fungi. Environ Sci Technol. 2005;39:4640-6.

45. Warmink JA, Nazir R, Corten B, van Elsas JD. Hitchhikers on the fungal highway: The helper effect for bacterial migration via fungal hyphae. Soil Biol Biochem. 2011;43:760-5.

46. Barka EA, Vatsa P, Sanchez L, Nathalie Gaveau-Vaillant CJ, Klenk H-P, Clément C, et al. Taxonomy, physiology, and natural products of Actinobacteria. Am Soc Microbiol. 2016;80:1-43.

47. Hess WM, Stocks DL. Surface characteristics of Aspergillus conidia. Mycologia. 1969;61:560-71.

48. Hess WM, Sassen MA, Remsen CC. Surface characteristics of Penicillium Conidia. Mycologia. 1968;60:290-303.

49. Kieser T, Bibb MJ, Chater KF, Butter MJ, Hopwood DA, Chater $\mathrm{KF}$, et al. Practical Streptomyces genetics. Norwich, UK: John Innes Foundation; 2000. p. 1-613.

50. Richter M, Willey JM, Süßmuth R, Jung G, Fiedler HP. Streptofactin, a novel biosurfactant with aerial mycelium inducing activity from Streptomyces tendae Tu 901/8c. FEMS Microbiol Lett. 1998;163:165-71.

51. Mastronarde DN. Dual-axis tomography: An approach with alignment methods that preserve resolution. J Struct Biol. 1997;120:343-52.

52. Xiong Q, Morphew MK, Schwartz CL, Hoenger AH, Mastronarde DN. CTF determination and correction for low dose tomographic tilt series. J Struct Biol. 2009;168:378-87.

53. Pham HT, Parkinson JS. Phenol sensing by Escherichia coli chemoreceptors: a nonclassical mechanism. J Bacteriol. 2011;193:6597-604. 